\title{
Evaluation of bovine zona pellucida characteristics in polarized light as a prognostic marker for embryonic developmental potential
}

\author{
M Koester, A Mohammadi-Sangcheshmeh ${ }^{1}$, M Montag, F Rings ${ }^{1}$, T Schimming ${ }^{2}$, D Tesfaye ${ }^{1}$, \\ K Schellander ${ }^{1}$ and $M$ Hoelker $^{1}$ \\ Department of Gynecological Endocrinology and Reproductive Medicine, University of Bonn, Sigmund-Freud-Strasse \\ 25, 53127 Bonn, Germany, ${ }^{1}$ Animal Breeding and Husbandry Group, Institute of Animal Science, University of Bonn, \\ Endenicher Allee 15, 53115 Bonn, Germany and ${ }^{2}$ Octax Microscience GmbH, Dr-Pauling-Strasse 9, 84079 \\ Bruckberg, Germany
}

Correspondence should be addressed to M Koester; Email: maria.koester@ukb.uni-bonn.de

\begin{abstract}
It has previously been demonstrated that zona pellucida imaging of human oocytes using polarized light microscopy is a clinically applicable method for the noninvasive assessment of oocyte quality. This study was designed to investigate whether zona pellucida characteristics of bovine oocytes and zygotes in polarized light may similarly serve as a useful marker for developmental competence in bovine reproductive biotechnologies. Zona birefringence intensity parameters of 2862 oocytes/zygotes were objectively evaluated with an automatic analysis system and correlated with oocyte/zygote quality. In detail, immature oocytes of good quality assessed with brilliant cresyl blue staining showed significantly lower zona birefringence than poor-quality counterparts $(P<0.001)$. After in vitro maturation and classification according to maturational status, the birefringence intensity parameters were significantly different in those oocytes that reached metaphase II compared with arrested stages $(P<0.001)$. Following either parthenogenetic activation or IVF with subsequent in vitro culture in a well-of-the-well system until day 9 , superior development as determined by cleavage, blastocyst formation, and hatching ability was associated with lower zona birefringence intensity parameters. When early zygote-stage embryos were selected and assorted in groups based on zona birefringence (high/medium/low), the group of embryos derived from high-birefringence zygotes displayed a significantly compromised developmental potential compared with low-birefringence zygotes. These results clearly show that developmentally competent bovine oocytes/zygotes exhibit lower zona birefringence intensity parameters. Therefore, birefringence imaging of zona pellucida is a suitable technique to predict bovine preimplantation embryo development.

Reproduction (2011) 141 779-787
\end{abstract}

\section{Introduction}

It is well known that both the maternal and paternal gametes are highly differentiated cells and they are the origin of each individual life. Whilst the spermatozoon is crucial for the initiation of fertilization and completion of the diploid chromosomal set, it is predominantly the quality of the oocyte that determines the subsequent development of the embryo. The backbone for this developmental competence of the oocyte is acquired through oogenesis. During that process, the oocyte is provided with the required cellular and molecular components, succeeding in the ability of being fertilized and capable of directing early preimplantation development. The necessity of a wellorchestrated oogenesis for later embryogenesis was already recognized at the beginning of the 20th century and is still in focus of current research. Although the molecular mechanisms are not yet fully understood, it is generally accepted that oocyte quality is a key factor for optimizing the efficiency of reproductive techniques in farm animal as well as for human-assisted reproductive technologies (ARTs, for review, see Coticchio et al. 2004, Sirard et al. 2006, van Soom et al. 2007, Telfer \& McLaughlin 2007, Wang \& Sun 2007).

Therefore, numerous attempts have been made to identify prognostic factors that may allow selection of oocytes according to their developmental potential. In cattle, follicle size along with oocyte diameter is traditionally considered as valuable parameter for oocyte maturity and, consequently, quality (Pavlok et al. 1992, Lonergan et al. 1994, Fair et al. 1995, Otoi et al. 1997). Another established, noninvasive method is 
the morphological assessment based on the homogeneity of ooplasm and thickness as well as compactness of surrounding cumulus layers (Gordon 1994, Blondin \& Sirard 1995, Nagano et al. 2006, Santos et al. 2008). However, the predictive value of morphological characteristics is controversially discussed, due to subjectivity, inaccuracy, and requirement of extensive experience (Wang \& Sun 2007, Nagy 2008).

Moreover, a variety of cellular and subcellular parameters have been investigated whether they are related to developmental competence, such as gene expression pattern (Wrenzycki et al. 2007), mitochondrial status (Stojkovic et al. 2001), calcium stores and calcium current activity (Boni et al. 2002), apoptotic index (Yuan et al. 2005), gene expression profiles in cumulus cells (Assidi et al. 2008, Tesfaye et al. 2009), and factors present in the follicular fluid (Nicholas et al. 2005, van Soom et al. 2007, Sinclair et al. 2008). Unfortunately, these techniques are often complex, timeconsuming, and, most importantly, invasive, which excludes further development of the oocyte/embryo.

A parameter of proven predictive value is assessing glucose-6-phosphate dehydrogenase (G6PDH) activity by brilliant cresyl blue (BCB) staining in immature cumulus oocyte complexes (COCs). BCB staining is an indicator for the stage of development expressed by the G6PDH activity without being harmful to the oocyte. Activity of G6PDH is high in growing oocytes because of the demand of ribose-6-phosphate for nucleotide synthesis and, correspondingly, low in those oocytes that have finished their growth phase (Mangia \& Epstein 1975). BCB staining has been successfully used as a diagnostic tool for oocyte evaluation in various species including cattle (Pujol et al. 2004, Alm et al. 2005, Bhojwani et al. 2007).

A new noninvasive method to investigate the developmental capacity of oocytes and zygotes has been proposed in human ART with the introduction of computer-assisted polarized light microscopy (Oldenbourg 1999, Liu et al. 2000). This rediscovered microscopic technique allows noninvasive visualization of subcellular structures that exhibit a natural birefringence due to a high molecular order in vital cells. The first structure that can be detected is the meiotic spindle, which is discussed as a predictor of oocyte quality (see meta-analysis by Petersen (2009)). A recently published comparison of spindle imaging in polarized light with high-performance confocal microscopy showed, however, that the identification of spindles in polarized light may give only limited information about the spindle constitution (Coticchio et al. 2010).

The second structure that can be visualized in polarized light is the zona pellucida. This extracellular glycoprotein coat surrounding all mammalian oocytes and preimplantation embryos is mainly formed from the oocyte during oogenesis (Wassarman 1988, Epifano et al. 1995, Green 1997, Sinowatz et al. 2001).
Hence, the texture of the zona pellucida might reflect the history of folliculogenesis (Pelletier et al. 2004) and the influence of in vitro culture (Keefe et al. 2003). Keefe et al. (1997) revealed the trilaminar architecture of hamster zona pellucida with the inner layer exhibiting the highest amount of birefringence. This was also observed in human oocytes (Pelletier et al. 2004). Retrospectively, a higher anisotropic inner layer was associated with significantly higher conception rates after embryo transfer compared with oocytes showing a lower retarding inner layer (Shen et al. 2005). Zona pellucida imaging at the oocyte stage was also successfully established as a predictive marker for human oocyte quality in several other studies (Rama Raju et al. 2007, Montag et al. 2008, Madaschi et al. 2009, Ebner et al. 2010). However, all published studies focused on human ART, and it remains an open question whether the results of these studies could be transferred to other species. To our knowledge, no studies have been performed so far that elucidate the properties of the bovine zona pellucida in polarized light and the suspected prognostic value according to developmental competence.

The aim of this study was, therefore, to evaluate the applicability of zona imaging to predict the developmental competence of oocytes and zygotes in the bovine species. For this, zona birefringence of immature and mature oocytes as well as early zygote stage embryos was examined in terms of different zona parameters and correlated with oocyte/zygote quality and developmental competence.

\section{Results}

A total of 2862 oocytes/zygotes were examined in polarized light with the automatic user-independent zona birefringence measuring system (Fig. 1).

\section{Experiment 1: correlation between zona pellucida birefringence and quality of immature bovine oocytes classified based on BCB stain}

When 836 immature oocytes were exposed to BCB stain, $406(48.6 \%)$ oocytes were classified as BCB + (high developmental potential). The proportion of colorless BCB - oocytes was $51.4 \%$, correspondingly (low developmental potential, $n=430$ ). As shown in Table 1, there was a clear difference between the two groups: mean values of cumulated value $(\mathrm{CV})$, peak value $(\mathrm{PV})$, and zona score were significantly lower in the BCB + oocytes.

\section{Experiment 2: correlation between zona pellucida birefringence and maturational stage of bovine oocytes matured in vitro}

When the meiotic configuration was determined after $22 \mathrm{~h}$ of in vitro maturation (IVM) for 446 oocytes, $75.6 \%$ 

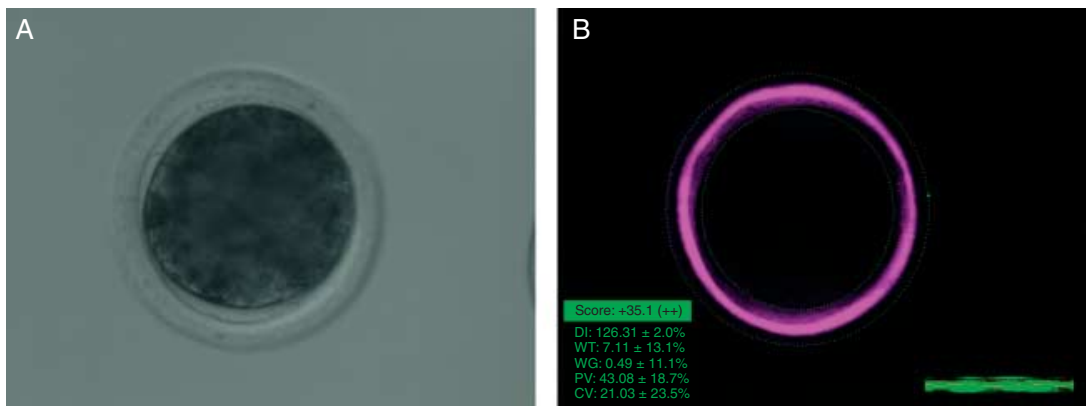

Figure 1 Imaging of an immature bovine oocyte by conventional light microscopy (A) and by polarized light microscopy (B).

of them $(n=337)$ had reached metaphase II. All investigated parameters regarding zona pellucida were significantly lower in the group of developing oocytes that had accomplished the appropriate meiotic stage compared with those that failed (Table 2).

\section{Experiment 3: correlation between zona pellucida birefringence and subsequent development of parthenogenetically activated bovine oocytes}

When 365 artificially activated oocytes were measured for zona properties immediately after activation and cultured individually up to day 9, average values for zona parameters of oocytes differed when comparing groups that reached different developmental stages. Activated oocytes that underwent cleavage had lower values for $\mathrm{CV}, \mathrm{PV}$, and zona score compared with oocytes that failed cleavage $(P<0.05)$. Similarly, parthenotes that reached the blastocyst stage had lower values compared with those that stopped development before. Finally, parthenotes that reached hatching blastocyst stage had lower average values for PV, CV, and zona score compared with activated oocytes that reached blastocyst stage but did not hatch. However, differences only reached statistical significance regarding $\mathrm{CV}$ value for blastocyst stage on day 7 (Table 3). The analyzed group did not differ from the control group in terms of cleavage, blastocyst, and hatching rate.

\section{Experiment 4: correlation between zona pellucida birefringence and subsequent development of in vitro fertilized bovine oocytes}

Focusing on developmental competence after IVF, there was an explicit association to zona values of PV and CV as well as the score. For all parameters investigated in a total of 415 zygotes, fertilized oocytes that cleaved until day 3 showed lower mean values than those that did not cleave at all. In addition, the difference between fertilized oocytes that reached blastocyst stage until day 7 in comparison to their counterparts that failed was highly significant. In a subgroup of day 7 blastocysts, namely expanded blastocysts, the zona of the corresponding fertilized oocytes expressed again significantly lower birefringence compared with the subgroup of non-expanded blastocysts. Moreover, hatched blastocysts on day 9 resulted from zygotes with lower zona values (Table 4). Compared with the control group, zona imaging had no effect regarding overall cleavage and blastocyst rate.

\section{Experiment 5: developmental rates of bovine zygotes after prospective classification according to zona pellucida birefringence}

In a final trial, we tested our previous retrospective results in a prospective experiment. Therefore, we allocated zygotes $(n=800)$ into three groups according to the zona score assessed in zygote stage. The group of zygotes with the lowest zona score (lowest 25\%) showed the highest developmental rates up to blastocyst stage. Among all groups, the lowest embryonic development was observed in the group of zygotes within the top 25\% for zona score. Moreover, hatching rate was higher for zygotes belonging to the lowest $75 \%$ compared with zygotes of the top $25 \%$ as well as for zygotes of the control group (Table 5).

\section{Discussion}

The identification of noninvasive prognostic markers for oocyte quality attracts widespread attention in assisted reproductive technologies. In human ART, zona pellucida imaging using polarization light microscopy has been introduced as a reliable method for the evaluation of developmental competence in vital oocytes (Shen et al. 2005, Montag \& van der Ven 2008, Montag et al. 2008, Ebner et al. 2010).

In this study, we analyzed the correlation between oocyte/zygote developmental potential and zona pellucida birefringence in Bos taurus. As the zona matrix is

Table 1 Correlation between bovine zona pellucida properties and glucose-6-phosphate dehydrogenase activity.

\begin{tabular}{lcccc}
\hline Group & Total $(n)$ & $\begin{array}{c}\text { CV value } \\
(x \pm \text { s.D. })\end{array}$ & $\begin{array}{c}\text { PV value } \\
(x \pm \text { s.D. })\end{array}$ & $\begin{array}{c}\text { Score } \\
(x \pm \text { s.D. })\end{array}$ \\
\hline BCB + & $48.6 \%(406 / 836)$ & $21.67 \pm 4.62^{\mathrm{a}}$ & $41.59 \pm 9.12^{\mathrm{c}}$ & $33.09 \pm 14.51^{\mathrm{e}}$ \\
$\mathrm{BCB}-$ & $51.4 \%(430 / 836)$ & $24.92 \pm 4.94^{\mathrm{b}}$ & $46.16 \pm 9.30^{\mathrm{d}}$ & $39.04 \pm 14.70^{f}$ \\
\hline a:b, c:d, e:f $P<0.001$. & & &
\end{tabular}


Table 2 Correlation between bovine zona pellucida properties and meiotic stage.

\begin{tabular}{lcccc}
\hline Meiotic stage & Total $(n)$ & CV value $(x \pm$ s.D. $)$ & PV value $(x \pm$ s.D. $)$ & Score $(x \pm$ s.D. $)$ \\
\hline Metaphase II & $75.6 \%(337 / 446)$ & $24.63 \pm 6.73^{\mathrm{a}}$ & $48.13 \pm 13.44^{\mathrm{c}}$ & $39.67 \pm 18.04^{\mathrm{e}}$ \\
Metaphase I/GV & $24.4 \%(109 / 446)$ & $28.99 \pm 7.33^{\mathrm{b}}$ & $56.52 \pm 14.77^{\mathrm{d}}$ & $51.80 \pm 20.57^{\mathrm{f}}$ \\
\hline
\end{tabular}

a:b, c:d, e:f $P<0.001$.

generated during mammalian follicular development predominantly by the oocyte, we hypothesized that normality of oocyte growth and cytoplasmic functionality is reflected in the zona pellucida structure and that measuring zona birefringence characteristics may serve as an indicator of its physical properties.

In several experiments, we retrospectively investigated the correlation between zona birefringence parameters and oocyte/zygote quality determined on the one hand by G6PDH activity in immature oocytes (exp. 1) and degree of maturity after IVM (exp. 2) on the other hand by preimplantive development following parthenogenetic activation (exp. 3) and IVF (exp. 4). The results of all these retrospective experiments showed that low mean values for zona birefringence parameters (PV, CV, and zona score) were related to superior oocyte/zygote quality and subsequently led to better preimplantation development following artificial activation or IVF. The retrospective outcome was ultimately tested in a final prospective experiment (exp. 5) in which zygote-stage embryos were separated into different prognostic groups according to their zona score. The group of zygotes with low zona score values had significantly higher cleavage rates, much higher blastocyst rates until day 7 , and much higher hatching rates $(P<0.05)$. Therefore, this study clearly confirmed our hypothesis that zona properties in polarized light uncover developmental competence of bovine oocyte and zygotes.

In accordance with our results, it has been reported that an exceedingly high zona birefringence intensity of human oocytes is negatively correlated to blastocyst formation (Jelinkova et al. 2007), and Iwayama et al. (2010) found a positive correlation between the hardness of human zona pellucida and zona birefringence. Although the molecular background of zona birefringence is not yet fully understood, it is tempting to speculate that an intense birefringence, associated with high calculated values, is caused by a very tight and well-ordered zona pellucida microtexture. A highly concentrated zona ultrastructure, exposing intense birefringence, impairs cumulus-oocyte interaction, diminishes sperm binding as well as sperm penetration during fertilization and thus may have a negative impact on success rates (Familiari et al. 2006).

Our results are in striking contrast with previous observations in human oocytes, in which a higher birefringence intensity of the inner zona layer was associated with better developmental potential (Rama Raju et al. 2007, Montag et al. 2008, Madaschi et al. 2009, Ebner et al. 2010). In general, we observed that the inner zona layer of bovine oocytes showed a much higher birefringence compared with human oocytes (data not shown). However, our experiments are not completely comparable with the results from human studies. In this study, we analyzed oocytes that were conventionally harvested by ex vivo aspiration of small antral follicles, which is a standard practice in bovine in vitro embryo production. Contrarily, human oocytes were routinely obtained from patients after controlled ovarian stimulation by transvaginal ovum pickup, so those studies have been performed with in vivo matured, hormone-substituted oocytes. The discrepant findings between the bovine and the human system may be explained by these differences, which might influence final oocyte maturation.

Several authors have explored the morphology of the zona pellucida by scanning electron microscopy and found a correlation to the grade of oocyte maturity. Whilst immature oocytes possess a relatively smooth and compact shape, a shift to a more mesh-like, spongy

Table 3 Correlation between zona pellucida properties and development of bovine parthenotes.

\begin{tabular}{|c|c|c|c|c|}
\hline Developmental competence & Total $(n)$ & CV value $(x \pm$ S.D. $)$ & PV value $(x \pm$ s.D. $)$ & Score $(x \pm$ s.D. $)$ \\
\hline \multicolumn{5}{|l|}{ Cleavage rate $\mathrm{d} 3$} \\
\hline Cleaved & $78.6 \%(287 / 365)$ & $22.07 \pm 4.16$ & $42.33 \pm 7.69$ & $31.25 \pm 9.91$ \\
\hline Not cleaved & $21.4 \%(78 / 365)$ & $23.06 \pm 5.05$ & $43.87 \pm 8.76$ & $33.24 \pm 11.86$ \\
\hline \multicolumn{5}{|l|}{ Blastocyst rate $d 7$} \\
\hline Blastocyst & $40.1 \%(115 / 287)$ & $21.39 \pm 3.94^{\mathrm{a}}$ & $41.50 \pm 7.42$ & $30.51 \pm 9.39$ \\
\hline No blastocyst & $59.9 \%(172 / 287)$ & $22.53 \pm 4.23^{b}$ & $42.88 \pm 7.82$ & $31.74 \pm 10.21$ \\
\hline \multicolumn{5}{|l|}{ Hatching rate $\mathrm{d} 9$} \\
\hline Hatched & $37.3 \%(50 / 134)$ & $21.19 \pm 4.08$ & $41.04 \pm 7.41$ & $29.41 \pm 8.75$ \\
\hline Not hatched & $62.7 \%(84 / 134)$ & $21.98 \pm 4.09$ & $42.51 \pm 7.67$ & $31.70 \pm 9.69$ \\
\hline
\end{tabular}

a:b $P<0.05$. 
Table 4 Correlation between zona pellucida properties and development of bovine zygotes.

\begin{tabular}{|c|c|c|c|c|}
\hline Developmental competence & Total $(n)$ & CV value $(x \pm$ s.D. $)$ & PV value ( $x \pm$ s.D.) & Score $(x \pm$ S.D. $)$ \\
\hline \multicolumn{5}{|l|}{ Cleavage rate $\mathrm{d} 3$} \\
\hline Cleaved & $80.5 \%^{\mathrm{a}}(334 / 415)$ & $25.28^{\mathrm{a}} \pm 6.00$ & $48.25^{\mathrm{a}} \pm 12.11$ & $38.71^{\mathrm{a}} \pm 15.08$ \\
\hline Not cleaved & $19.5 \%{ }^{\mathrm{b}}(81 / 415)$ & $27.38^{\mathrm{b}} \pm 6.89$ & $51.79^{b} \pm 13.37$ & $43.73^{b} \pm 16.87$ \\
\hline \multicolumn{5}{|l|}{ Blastocyst rate $\mathrm{d} 7$} \\
\hline Blastocyst & $32.3 \%(108 / 334)$ & $23.72^{g} \pm 5.72$ & $44.93^{g} \pm 11.09$ & $34.38^{\mathrm{g}} \pm 13.18$ \\
\hline No blastocyst & $67.7 \%(226 / 334)$ & $26.03^{\mathrm{h}} \pm 5.98$ & $49.83^{\mathrm{h}} \pm 12.26$ & $40.77^{\mathrm{h}} \pm 15.49$ \\
\hline \multicolumn{5}{|l|}{ Expansion d7 } \\
\hline Expanded & $29.6 \%(32 / 108)$ & $21.39^{\mathrm{C}} \pm 3.80$ & $40.75^{\mathrm{C}} \pm 8.02$ & $30.02^{\mathrm{C}} \pm 10.16$ \\
\hline Not expanded & $70.4 \%(76 / 108)$ & $24.69^{\mathrm{d}} \pm 6.10$ & $46.69^{\mathrm{d}} \pm 11.72$ & $36.21^{\mathrm{d}} \pm 13.85$ \\
\hline \multicolumn{5}{|l|}{ Hatching rate d9 } \\
\hline Hatched & $52.0 \%(102 / 196)$ & $23.66^{\mathrm{e}} \pm 5.67$ & $44.28^{\mathrm{e}} \pm 10.98$ & $32.83^{\mathrm{e}} \pm 13.45$ \\
\hline Not hatched & $48.0 \%(94 / 196)$ & $25.79^{f} \pm 6.15$ & $48.01^{\mathrm{f}} \pm 11.81$ & $37.51^{\mathrm{f}} \pm 14.75$ \\
\hline
\end{tabular}

a:b, c:d, e:f $P<0.05 ;{ }^{\text {g:h }} P<0.001$.

appearance with numerous pores was described after maturation. The spongy network is probably necessary for the cytoplasmic protrusions from surrounding corona radiata cells, essential for cell-to-cell communication between the oocyte and the enclosing cumulus cells (Vanroose et al. 2000, Familiari et al. 2006, Santos et al. 2008). Furthermore, a correlation between the oocyte maturation stage and the zona binding properties of spermatozoa in the human and the rhesus monkey was reported (Oehninger et al. 1991, Pu et al. 1994). Interestingly, the relationship between the maturational state and the zona microtexture has lately been confirmed by polarization light microscopy. Compared with metaphase II stages, human immature oocytes express a higher zona birefringence (Cheng et al. 2010, Braga et al. 2010), and in this study, the same was found for bovine oocytes. So together with our results, there is more and more evidence that during maturation morphological and physical zona properties change from a rather compact structure with high birefringence to a more porous one exhibiting lower birefringence.

All these data indirectly point to significant modifications in the ultrastructure of the zona pellucida during the final maturation process from germinal vesicle to metaphase II stage. Whether this process is comparable in oocytes matured in vitro versus in vivo is not clarified yet. Definitely, IVM of bovine oocytes is associated with a longer exposure time in culture medium. There is no doubt that in the bovine, in vitro culture conditions have an influence on the developmental potential of the oocyte, and the ongoing development (Lonergan et al.
2003, Van Soom et al. 2007). Braga et al. (2010) recently observed no significant change in the percentages of high birefringence oocytes after spontaneous rescue maturation in vitro, which may indicate that completion of nuclear maturation in vitro is not synchronous with cytoplasmic maturation. Moreover, the effect of gonadotropin application on oocyte quality is subject of continuing debate, because recruitment of follicles is altered in a non-physiological way. Ebner et al. (2010) reported differences in zona birefringence intensity between stimulation protocols, and further studies are needed for a better understanding.

Apart from the different origin of examined oocytes, another possible explanation for the contradictory observations is that for human ART treatment, zona with higher birefringence intensity could be of advantage in ICSI as the oocyte might be protected particularly well from mechanical stress during the microinjection procedure and stress-related changes during preimplantation development, which might, in contrast, be detrimental for parthenogenetic activation or normal fertilization of bovine oocytes as performed in our study.

In conclusion, the data of this study show that for a bovine in vitro production programme, zona pellucida imaging by polarization light microscopy is a powerful tool to select oocytes of high developmental competence or to predict the developmental potential in immature and matured oocytes/zygotes. The findings of this study will be of great help for further research and could increase the quality of scientific studies dealing with bovine in vitro produced embryos by decreasing the

Table 5 Developmental rates of bovine zygotes after classification by zona score.

\begin{tabular}{|c|c|c|c|c|}
\hline Zona score & High score & Average score & Low score & Control \\
\hline$n$ & 205 & 399 & 196 & 311 \\
\hline Cleavage rate $\mathrm{d} 3$ & $67.8 \% \%^{a}(139 / 205)$ & $79.9 \%$ b $(319 / 399)$ & $83.1 \%{ }^{\mathrm{c}}(163 / 196)$ & $70.1 \%{ }^{d}(218 / 311)$ \\
\hline Blastocyst rate $\mathrm{d} 7$ & $13.2 \% \mathrm{e}(27 / 205)$ & $22.8 \%^{f}(91 / 399)$ & $24.5 \% \mathrm{~g}(48 / 196)$ & $18.3 \%(57 / 311)$ \\
\hline Blastocyst rate $\mathrm{d} 9$ & $17.6 \%{ }^{\mathrm{h}}(36 / 205)$ & $30.3 \%{ }^{\mathrm{i}}(121 / 399)$ & $32.7 \% \%^{j}(64 / 196)$ & $29.6 \%{ }^{k}(92 / 311)$ \\
\hline Hatching rate $\mathrm{d} 9$ & $7.8 \% \mathrm{l}(16 / 205)$ & $17.0 \%{ }^{\mathrm{m}}(68 / 399)$ & $16.3 \%^{\mathrm{n}}(32 / 196)$ & $10.6 \%^{\circ}(33 / 311)$ \\
\hline
\end{tabular}

a:b, a:c, c:d, h:i, h:j $P<0.001$; b:d, e:f, e:g, h:k, l:m, l:n, m:o $P<0.005$. 
developmental variability due to large differences in oocyte quality. Moreover, this technique may offer a great benefit in the identification of high-quality oocytes for the use in time-consuming techniques like nuclear transfer and ICSI. However, further studies are necessary to test the predictive value of optical zona properties for implantation efficiency, pregnancy rates, and calf delivery rates as well as calf health. In addition, analysis of in vitro versus in vivo matured oocytes using zona imaging would contribute to a better understanding of oocyte cytoplasmic maturation.

\section{Materials and Methods}

Unless stated, all biochemicals were purchased from Sigma.

\section{Collection of immature COCs}

Bovine ovaries were obtained from a local abattoir and transported to the laboratory within $3 \mathrm{~h}$ in physiological saline solution. While maintaining the temperature at $35-38{ }^{\circ} \mathrm{C}$, ovaries were rinsed three times. Follicular fluid was collected by aspiration of small follicles (ø 2-8 $\mathrm{mm}$ ) using an 18-gauge needle attached to a $10 \mathrm{ml}$ syringe. COCs were pooled under a stereomicroscope in HEPES-buffered tissue culture medium (TCM 199 with Earle's salts containing L-glutamine and $25 \mathrm{mmol} / \mathrm{l}$ HEPES) supplemented with $0.1 \%$ BSA, $22 \mu \mathrm{g} / \mathrm{ml}$ pyruvate, $350 \mu \mathrm{g} / \mathrm{ml} \mathrm{NaHCO}_{3}$, and $50 \mu \mathrm{g} / \mathrm{ml}$ gentamicin (Gibco BRL). Only those oocytes having a homogenous, evenly granulated ooplasm and possessing at least three layers of compact cumulus cells were selected and used in the experiments.

\section{Assessment of G6PDH activity by BCB staining}

The procedure of BCB staining was carried out according to a modified protocol described by Alm et al. (2005). Immature COCs of morphologically good quality were washed three times in Dulbecco's PBS supplemented with $26 \mu \mathrm{M} \mathrm{BCB}$ and $0.4 \%$ BSA and incubated in $400 \mu \mathrm{l}$ staining solution under mineral oil for $30 \mathrm{~min}$ at $38^{\circ} \mathrm{C}$. After washing in Dulbecco's PBS $\left(\mathrm{Ca}^{2+}, \mathrm{Mg}^{2+}\right.$ free, and D-PBS), the stained COCs were examined under a stereomicroscope and classified into two groups according to their ooplasm coloration. In oocytes that exhibit a colorless ooplasm, BCB is metabolized rapidly; these oocytes have not yet reached developmental competence $(\mathrm{BCB}-)$. In oocytes with various degrees of blue coloration, the blue-colored cytoplasm is a marker for a reduced enzyme activity; those oocytes are likely to be developmentally competent (BCB +; Ericsson et al. 1993). For denudation and zona imaging, somatic cells were removed both mechanically and enzymatically by vortexing in $500 \mu$ l bovine hyaluronidase $(1 \mathrm{mg} / \mathrm{ml}$ in D-PBS) for $3 \mathrm{~min}$.

\section{IVM of bovine oocytes}

After three washes, morphologically selected immature COCs were transferred in groups of 50 into $400 \mu$ of maturation medium covered with mineral oil. TCM 199 with Earle's salts served as basic medium and was modified with $100 \mu \mathrm{g} / \mathrm{ml}$ L-glutamine, $250 \mu \mathrm{g} / \mathrm{ml}$ pyruvate, $600 \mu \mathrm{g} / \mathrm{ml}$ hemicalcium lactate, $800 \mu \mathrm{g} / \mathrm{ml} \mathrm{NaHCO}, 1.4 \mathrm{mg} / \mathrm{ml}$ HEPES, $50 \mu \mathrm{g} / \mathrm{ml}$ gentamicin, $12 \%$ heat-inactivated estrus cow serum (OCS), and $10 \mu \mathrm{g} / \mathrm{ml} \mathrm{FSH}$ (Follitropin, Vetrepharm, Ireland). IVM was performed in 4-well dishes (Nunc, Roskilde, Denmark) in humified atmosphere composed of $5 \% \mathrm{CO}_{2}$ in air at $39{ }^{\circ} \mathrm{C}$ for $22 \mathrm{~h}$.

\section{Parthenogenetic activation of bovine oocytes}

Following IVM, oocytes were vortexed in $500 \mu$ l hyaluronidase ( $1 \mathrm{mg} / \mathrm{ml}$ in D-PBS) for $3 \mathrm{~min}$ to remove the surrounding cumulus cells. Denuded oocytes with intact oolemma were washed twice and subsequently activated by a two-step procedure. The first step was an incubation in $5 \mu \mathrm{M}$ ionomycin (in HEPES-modified TCM 199 as described earlier) for $4 \mathrm{~min}$. Oocytes were then rinsed in culture medium (CR1 aa, Rosenkrans \& First 1994) and incubated in the same medium supplemented with $2 \mathrm{mM}$ 6-dimethylaminopurine (DMAP) for $3.5 \mathrm{~h}$ at $39{ }^{\circ} \mathrm{C}$ in $5 \% \mathrm{CO}_{2}$ in air with maximum humidity. Activated oocytes were washed three times in CR1 aa medium. Before culturing the individual in a modified well-of-the-well (WOW) dish, zona imaging was performed (see culture methods below).

\section{IVF of bovine oocytes}

For IVF, frozen semen from one bull with proven fertility in IVF was prepared using a modified swim up technique (Parrish et al. 1988). Briefly, frozen straws were thawed at a temperature of $39.5^{\circ} \mathrm{C}$ for $10 \mathrm{~s}$ in a water bath. Motile sperm were separated by swim up for $50 \mathrm{~min}$ in Sperm-TALP containing $6 \mathrm{mg} / \mathrm{ml} \mathrm{BSA} \mathrm{(Parrish} \mathrm{et} \mathrm{al.} \mathrm{1988)} \mathrm{and} \mathrm{centrifuged}$ at $250 \mathrm{~g}$ for $10 \mathrm{~min}$. The pellet was resuspended in Fert-TALP supplemented with $20 \mu \mathrm{M}$ penicillamine, $10 \mu \mathrm{M}$ hypotaurine, $2 \mu \mathrm{M}$ epinephrine, $2 \mu \mathrm{g} / \mathrm{ml}$ heparin, $6 \mathrm{mg} / \mathrm{ml} \mathrm{BSA}, 2.2 \mathrm{mg} / \mathrm{ml}$ pyruvate, and $50 \mu \mathrm{g} / \mathrm{ml}$ gentamicin. In vitro matured COCs were placed into $400 \mu \mathrm{l}$ of modified Fert-TALP in 4-well dishes covered with mineral oil. Final concentration of spermatozoa in each well was adjusted to $2 \times 10^{6} \mathrm{million} / \mathrm{ml}$. Gametes were coincubated for $19 \mathrm{~h}$ under the same temperature and gas conditions as described for IVM. Fertilized oocytes were denuded mechanically by vortexing in HEPES-modified TCM and rinsed in culture medium CR1aa. After zona imaging, presumptive zygotes were cultured in a WOW dish (see culture methods below).

\section{In vitro culture of bovine oocytes}

In vitro embryo development was performed in CR1 aa culture medium (Rosenkrans \& First 1994) containing 10\% heatinactivated OCS, and essential and non-essential amino acids (MEM, BME; Gibco BRL) at $39{ }^{\circ} \mathrm{C}$ under humidified atmosphere of $5 \% \mathrm{CO}_{2}$ in air. In order to individually monitor embryo development, artificially activated oocytes as well as presumptive zygotes were cultured in a modified WOW system (Vatja et al. 2000). Miniwells were prepared in 5-well dishes (Minitüb, Tiefenbach, Germany) as described recently (Hoelker 
et al. 2009). Briefly, a total of 16 small holes with depth and diameter of $0.7 \mathrm{~mm}$ were drilled in the bottom of each well. Modified wells were flushed with culture medium to remove air bubbles and possible toxic material. Then, $400 \mu \mathrm{l}$ of culture medium was overlaid with $400 \mu \mathrm{l}$ mineral oil and zona-imaged presumptive embryos were carefully transferred in each miniwell. When culture period was accomplished in group, 50 embryos were cultured together in one well of $400 \mu \mathrm{l}$ culture medium covered with mineral oil.

\section{Assessment of developmental competence}

Cleavage of embryos and parthenotes was recorded $72 \mathrm{~h}$ after placing into culture. On days 7 and 9, further development to blastocyst stage and hatching was evaluated.

\section{Zona pellucida life imaging of bovine oocytes}

The technical setup for live zona imaging was an inverted microscope (Nikon Eclipse TE-2000; Nikon, Düsseldorf, Germany) equipped with $\times 20$ Hoffmann interference optics, a circular polarization filter, liquid crystal analyzer optics, and a fully heated ceramic plate with a glass insert in the objective pathway (at $38.5 \pm 0.5^{\circ} \mathrm{C}$ ). A polarization imaging software module (OCTAX polarAIDE implemented in OCTAX eyeware; MTG, Altdorf, Germany) enabled automatic analysis of zona birefringence. In detail, the image processing on the birefringence image extracted several birefringence intensity profiles $(n>20)$ across the inner zona layer. For each of the profiles, i.e. along the entire zona layer, the local birefringence intensity peak value PV and a cumulated birefringence value CV over the inner zona layer's width were calculated. These values (which were computed for each intensity profile, i.e. over the entire cell's circumference) were averaged and resulted in the values PV-Mean and CV-Mean. Furthermore, all statistics were combined into a single-score value using a patented algorithm that had previously been optimized to maximally correlate with a human oocyte's developmental potential (Montag \& van der Ven 2008, Montag et al. 2008, Ebner et al. 2010). Details of these calculations can be found in the European patent description EP 1918692 (Schimming \& Rink 2011).

As plastic dishes interfere with polarized light, glass bottom dishes (WillCo, Amsterdam, The Netherlands) were used for examination. Denuded oocytes/zygotes were separated in $4 \mu \mathrm{l}$ drops of HEPES-modified TCM (see above) and imaged at $200 \times$ magnification. Each dish contained 16 drops which were covered with mineral oil; oocyte/zygote screening did not last longer than 2 min per dish. Presumptive zygotes were examined in culture medium in the same way.

\section{Statistical analysis}

Developmental rates of embryos generated by parthenogenetic activation and IVF were analyzed by $\chi^{2}$ test. ANOVA (twotailed $t$-test) was performed for comparison of mean values of zona pellucida evaluation. Differences of $P \leq 0.05$ were considered to be statistically significant.

\section{Experimental design}

Experiment 1: correlation between zona pellucida birefringence and quality of immature bovine oocytes classified by BCB-stain

As a basic experiment, zona birefringence of immature oocytes was analyzed. In order to investigate a possible correlation between zona birefringence and oocyte quality, a cohort of COCs was subjected to BCB-staining and classified into $\mathrm{BCB}^{+}$- and $\mathrm{BCB}^{-}$groups prior to zona imaging. Values of PV-Mean, CV-Mean, and zona score were compared between the two groups.

Experiment 2: correlation between zona pellucida birefringence and maturational stage of bovine oocytes matured in vitro

Following IVM, oocytes were examined in polarized light. Each oocyte was rotated gently with the use of an injection pipette to identify the first polar body and the occurrence of a meiotic spindle. Only oocytes with a visible first polar body were graded as metaphase II. Meanwhile, zona birefringence parameters were recorded.

Experiment 3: correlation between zona pellucida birefringence and subsequent development of parthenogenetically activated bovine oocytes

To evaluate whether zona birefringence has a predictive value for developmental competence, in vitro matured oocytes were parthenogenetically activated prior to zona imaging and cultured individually for a period of 9 days. Values of PV-Mean, CV-Mean, and zona score were investigated between cleaved and non-cleaved activated oocytes. In addition, parthenotes that progressed to blastocyst stage on day 7 were compared to those that failed, and on day 9, hatching ability was correlated to zona birefringence. Artificially activated oocytes cultured in vitro in groups of 50 served as controls.

\section{Experiment 4: correlation between zona pellucida birefringence and subsequent development of in vitro fertilized bovine oocytes}

In this experiment, zona imaging had to be performed after IVF, as examination of zona pellucida in polarized light is only possible with denuded oocytes/zygotes. Presumptive zygotes were screened $21 \mathrm{~h}$ post insemination and further embryonic development was recorded individually until day 9. Data were analyzed in the same way as in experiment 3. In addition, zygotes progressed to expanded blastocyst stage on day 7 were compared with those that developed to non-expanded blastocyst stage. Fertilized oocytes cultured in groups of 50 after IVF were used as control group.

Experiment 5: developmental rates of bovine zygotes after prospective classification according to zona pellucida birefringence

In order to verify the results of the previous experiments, in vitro matured oocytes were fertilized in vitro and individual zona score of 200 presumptive zygotes per experiment was 
determined $21 \mathrm{~h}$ post insemination. Then, zygotes were classified into three groups according to their zona score: the first group contained the 50 zygotes with the highest zona score (>41.0 in average); approximately, 100 zygotes with a midrange score (25.5-41.0) belonged to group 2 and those 50 zygotes with the lowest score were selected as group $3(<25.5$ in average). During in vitro culture in 4-wells, cleavage rate, blastocyst rate on days 7 and 9 , and hatching rate on day 9 were assessed. Embryonic development of presumptive zygotes not undergoing zona imaging served as a control.

\section{Declaration of interest}

T Schimming is working for Octax Microscience $\mathrm{GmbH}$ which is the company producing the zona-imaging system used in this work. All other authors declare that there is no conflict of interest that could be perceived as prejudicing the impartiality of the research reported.

\section{Funding}

This research did not receive any specific grant from any funding agency in the public, commercial, or not-for-profit sector.

\section{References}

Alm H, Torner H, Löhrke B, Viergutz T, Ghoneim IM \& Kanitz W 2005 Bovine blastocyst development rate in vitro is influenced by selection of oocytes by brilliant cresyl blue staining before IVM as indicator for glucose-6-phosphate dehydrogenase activity. Theriogenology 63 2194-2205. (doi:10.1016/j.theriogenology.2004.09.050)

Assidi M, Dufort I, Ali A, Hamel M, Algriany O, Dielemann S \& Sirard MA 2008 Identification of potential markers of oocyte competence expressed in bovine cumulus cells matured with follicle-stimulating hormone and/or phorbol myristate acetate in vitro. Biology of Reproduction $\mathbf{7 9}$ 209-222. (doi:10.1095/biolreprod.108.067686)

Bhojwani S, Alm H, Torner H, Kanitz W \& Poehland R 2007 Selection of developmentally competent oocytes through brilliant cresyl blue stain enhances blastocyst development rate after bovine nuclear transfer. Theriogenology 67 341-345. (doi:10.1016/j.theriogenology.2006. 08.006)

Blondin P \& Sirard M 1995 Oocyte and follicular morphology as determining characteristics for developmental competence in bovine oocytes. Molecular Reproduction and Development 41 54-62. (doi:10. 1002/mrd.1080410109)

Boni R, Cuomo A \& Tosti E 2002 Developmental potential in bovine oocytes is related to cumulus-oocyte complex grade, calcium current activity, and calcium stores. Biology of Reproduction 66 836-842. (doi:10.1095/ biolreprod66.3.836)

Braga D, Figueira R, Queiroz P, Madaschi C, laconelli A \& Borges E 2010 Zona pellucida birefringence in in vivo and in vitro matured oocytes. Fertility and Sterility 94 2050-2053. (doi:10.1016/j.fertnstert.2009.12. 005)

Cheng J, Huang L, He B, Lu F, Wang X, Wu Z \& Shi D 2010 Quantitative analysis of the intensity of zona pellucida birefringence of oocytes during IVF cycles. Reproduction, Fertility, and Development 22 1167-1174. (doi:10.1071/RD10047)

Coticchio G, Sereni E, Serrao L, Mazzone S, ladarola I \& Borini A 2004 What criteria for the definition of oocyte quality? Annals of the New York Academy of Sciences 1034 132-144. (doi:10.1196/annals.1335.016)

Coticchio G, Sciajno R, Hutt K, Bromfield J, Borini A \& Albertini DF 2010 Comparative analysis of the metaphase II spindle of human oocytes through polarized light and high-performance confocal microscopy. Fertility and Sterility 93 2056-2064. (doi:10.1016/j.fertnstert.2008. 12.011)
Ebner T, Balaban B, Moser M, Shebl O, Urman B, Ata B \& Tews G 2010 Automatic user-independent zona pellucida imaging at the oocyte stage allows for the prediction of preimplantation development. Fertility and Sterility 94 913-920. (doi:10.1016/j.fertnstert.2009.03.106)

Epifano O, Liang LF, Familiari M, Moos MC \& Dean J 1995 Coordinate expression of the three zona pellucida genes during mouse oogenesis. Development 121 1947-1956.

Ericsson S, Boice M, Funahashi H \& Day B 1993 Assessment of porcine oocytes using brilliant cresyl blue. Theriogenology 39 214. (doi:10.1016/ 0093-691X(93)90069-H)

Fair T, Hyttel P \& Greve T 1995 Bovine oocyte diameter in relation to maturational competence and transcriptional activity. Molecular Reproduction and Development 42 437-442. (doi:10.1002/mrd.1080 420410)

Familiari G, Relucenti M, Heyn R, Micara G \& Correr S 2006 Threedimensional structure of the zona pellucida at ovulation. Microscopic Research and Technique 69 415-426. (doi:10.1002/jemt.20301)

Gordon I 1994 Oocyte recovery and maturation. In Laboratory Production of Cattle Embryos, pp 30-142. Wallingford: $C A B$ International Press.

Green DP 1997 Three-dimensional structure of the zona pellucida. Reviews of Reproduction 2 147-156. (doi:10.1530/ror.0.0020147)

Hoelker M, Rings F, Lund Q, Ghanem N, Phatsara C, Griese J, Schellander K \& Tesfaye D 2009 Effect of the microenvironment and embryo density on developmental characteristics and gene expression profile of bovine preimplantative embryos cultured in vitro. Reproduction 137 415-425. (doi:10.1530/REP-08-0370)

Iwayama H, Hochi S \& Yamashita M 2010 Birefringence parameter available for quantitative analysis of human zona hardness. Zygote $\mathbf{2 7}$ 1-7. (doi:10.1017/S0967199410000389)

Jelinkova L, Reeka N, Heine A \& Gagsteiger F 2007 Correlation of automatic evaluation of the zona pellucida using polaraide ZP-score and embryo morphology on day 5. Journal of Reproductive Medicine and Endocrinology 5 P41.

Keefe D, Tran P, Pellegrini C \& Oldenburg R 1997 Polarized light microscopy and digital image processing identify a multilaminar structure of the hamster zona pellucida. Human Reproduction 12 1250-1252. (doi:10.1093/humrep/12.6.1250)

Keefe D, Liu L, Wang W \& Silva C 2003 Imaging meiotic spindles by polarization light microscopy: principles and applications to IVF. Reproductive Biomedicine Online 7 24-29. (doi:10.1016/S14726483(10)61724-5)

Liu L, Oldenbourg R, Trimarchi JR \& Keefe DL 2000 A reliable, noninvasive technique for spindle imaging and enucleation of mammalian oocytes. Nature Biotechnology 18 223-225. (doi:10.1038/72692)

Lonergan P, Monaghan P, Rizos D, Boland MP \& Gordon I 1994 Effect of follicle size on bovine oocyte quality and developmental competence following in vitro maturation, fertilization and culture in vitro. Molecular Reproduction and Development 37 48-53. (doi:10.1002/mrd.1080 370107)

Lonergan P, Rizos D, Gutierrez-Adan A, Fair T \& Boland MP 2003 Oocyte and embryo quality: effect of origin, culture condidions and gene expression patterns. Reproduction in Domestic Animals 38 259-267. (doi:10.1046/j.1439-0531.2003.00437.x)

Madaschi C, Aoki T, Braga D, Figueira R, Semiao Francisco L, laconelli A \& Borges E 2009 Zona pellucida birefringence score and meiotic spindle visualization in relation to embryo development and ICSI outcome. Reproductive Biomedicine Online 18 681-686. (doi:10.1016/S14726483(10)60014-4)

Mangia F \& Epstein C 1975 Biochemical studies of growing mouse oocytes: preparation of oocytes and analysis of glucose-6-phosphate dehydrogenase and lactate dehydrogenase activities. Developmental Biology 45 211-220. (doi:10.1016/0012-1606(75)90061-5)

Montag M \& van der Ven H 2008 Oocyte assessment and embryo viability prediction: birefringence imaging. Reproductive Biomedicine Online 17 454-460. (doi:10.1016/S1472-6483(10)60231-3)

Montag M, Schimming T, Köster M, Zhou C, Dorn C, Rösing B, van der Ven H \& van der Ven K 2008 Oocyte zona birefringence intensity is associated with embryonic implantation potential in ICSI cycles. Reproductive Biomedicine Online 16 239-244. (doi:10.1016/S14726483(10)60580-9) 
Nagano M, Katagiri S \& Takahashi Y 2006 Relationship between bovine oocyte morphology and in vitro developmental potential. Zygote 14 53-61. (doi:10.1017/S0967199406003510)

Nagy ZP 2008 Symposium: innovative techniques in human embryo viability assessment. Introduction. Reproductive Biomedicine Online 17 451-453. (doi:10.1016/S1472-6483(10)60230-1)

Nicholas B, Alberio R, Fouladi-Nashta AA \& Webb R 2005 Relationship between low-molecular-weight insulin-like growth factor-binding proteins, caspase-3 activity, and oocyte quality. Biology of Reproduction 72 796-804. (doi:10.1095/biolreprod.104.036087)

Oehninger S, Veeck L, Franken D, Kruger TF, Acosta AA \& Hodgen GD 1991 Human preovulatory oocytes have a higher sperm-binding ability than immature oocytes under hemizona assay conditions: evidence supporting the concept of "zona maturation". Fertility and Sterility 55 1165-1170.

Oldenbourg R 1999 Polarized light microscopy of spindles. Methods in Cell Biology 61 175-208.

Otoi T, Yamamoto K, Koyama N, Tachikawa S \& Suzuki T 1997 Bovine oocyte diameter in relation to developmental competence. Theriogenology 48 769-774. (doi:10.1016/S0093-691X(97)00300-2)

Parrish J, Susko-Parrish J, Winer M \& First N 1988 Capacitation of bovine sperm by heparin. Biology of Reproduction 38 1171-1180. (doi:10. 1095/biolreprod38.5.1171)

Pavlok A, Lucas-Hahn A \& Niemann H 1992 Fertilization and developmental competence of bovine oocytes derived from different categories of antral follicles. Molecular Reproduction and Development 31 63-67. (doi:10.1002/mrd.1080310111)

Pelletier C, Keefe DL \& Trimarchi JR 2004 Noninvasive polarized light microscopy quantitatively distinguishes the multilaminar structure of the zona pellucida of living human eggs and embryos. Fertility and Sterility 81 850-856. (doi:10.1016/j.fertnstert.2003.09.033)

Petersen C 2009 Relationship between visualization of meitotic spindle in human oocytes and ICSI outcomes: a meta-analysis. Reproductive Biomedicine Online 18 235-243. (doi:10.1016/S1472-6483(10)60261-1)

Pu XC, Ji WZ, Yang SC, Chen JC, Zou RJ \& Shang EY 1994 Correlation of zona-binding with oocyte maturation and sperm motility in rhesus monkeys by hemizona assay. Molecular Reproduction and Development 39 25-29. (doi:10.1002/mrd.1080390105

Pujol M, Lopez-Bejar M \& Paramio MT 2004 Developmental competence of heifer oocytes selected using the brilliant cresyl blue (BCB) test. Theriogenology 61 735-744. (doi:10.1016/S0093-691X(03)00250-4)

Rama Raju GA, Prakash GJ, Murali Krishna K \& Madan K 2007 Meiotic spindle and zona pellucida characteristics as predictors of embryonic development: a preliminary study using polscope imaging. Reproductive Biomedicine Online 14 166-174. (doi:10.1016/S1472-6483(10)60784-5)

Rosenkrans J \& First N 1994 Effect of free amino acids and vitamins on cleavage and developmental rate of bovine zygotes in vitro. Journal of Animal Science 72 434-437.

Santos P, Chaveiro A, Simoes N \& Moreira da Silva F 2008 Bovine oocyte quality in relation to ultrastructural characteristics of zona pellucida, polyspermic penetration and developmental competence. Reproduction in Domestic Animals 43 685-689. (doi:10.1111/j.1439-0531.2007. 00970.x)

Schimming T \& Rink K 2011 Method and computer program product for defining anisotropy parameters. European patent EP1918692. http:// www.freepatentsonline.com/EP1918692.html.

Shen Y, Stalf T, Mehnert C, Eichenlaub-Ritter U \& Tinneberg HR 2005 High magnitude of light retardation by the zona pellucida is associated with conception cycles. Human Reproduction 20 1596-1606. (doi:10.1093/ humrep/deh811)
Sinclair KD, Lunn LA, Kwong WY, Wonnacott K, Linforth RS \& Craigon J 2008 Amino acid and fatty acid composition of follicular fluid as predictors of in-vitro embryo development. Reproductive Biomedicine Online 16 859-868. (doi:10.1016/S1472-6483(10)60153-8)

Sinowatz F, Töpfer-Petersen E, Kolle S \& Palma G 2001 Functional morphology of the zona pellucida. Anatomia, Histologia, Embryologia 30 257-263. (doi:10.1046/j.1439-0264.2001.00337.x)

Sirard M, Richard F, Blondin P \& Robert C 2006 Contribution of the oocyte to embryo quality. Theriogenology 65 126-136. (doi:10.1016/j.theriogenology.2005.09.020)

Stojkovic M, Machado SA, Stojkovic P, Zakhartchenko V, Hutzler P, Goncalves PB \& Wolf E 2001 Mitochondrial distribution and adenosine triphosphate content of bovine oocytes before and after in vitro maturation: correlation with morphological criteria and development capacity after in vitro fertilization and culture. Biology of Reproduction 64 904-909. (doi:10.1095/biolreprod64.3.904)

Telfer E \& McLaughlin M 2007 Natural history of the mammalian oocyte. Reproductive Biomedicine Online 15 288-295. (doi:10.1016/S14726483(10)60341-0)

Tesfaye D, Ghanem N, Carter F, Fair T, Sirard MA, Hoelker M, Schellander K \& Lonergan P 2009 Gene expression profile of cumulus cells derived from cumulus-oocyte complexes matured either in vivo or in vitro. Reproduction, Fertility, and Development 21 451-461. (doi:10. 1071/RD08190)

Vanroose G, Nauwynck H, Van Soom A, Ysebaert M, Charlier G, Van Oostveldt P \& De Kruif A 2000 Structural aspects of the zona pellucida of in vitro-produced bovine embryos: a scanning electron and convocal laser scanning microscopic study. Biology of Reproduction 62 463-469. (doi:10.1095/biolreprod62.2.463)

Van Soom A, Vandaele L, Goossens K, de Kruif A \& Peelman L 2007 Gamete origin in relation to early embryo development. Theriogenology $\mathbf{6 8}$ S131-S137. (doi:10.1016/j.theriogenology.2007.03.019)

Vatja G, Peura TT, Holm P, Paldi A, Greve T, Trounson AO \& Callesen H 2000 New method for culture of zona-included or zona-free embryos: the well of the well (WOW) system. Molecular Reproduction and Development 55 256-264. (doi:10.1002/(SICl)1098-2795(200003)55:3 $<256$ ::AID-MRD3>3.0.CO;2-7)

Wang Q \& Sun QY 2007 Evaluation of oocyte quality: morphological, cellular and molecular predictors. Reproduction, Fertility, and Development 19 1-12. (doi:10.1071/RD06103)

Wassarman M 1988 The mammalian ovum. In The Physiology of Reproduction, pp 69-102. Eds E Knobil\& JD Neil. New York: Raven Press.

Wrenzycki C, Herrmann D \& Niemann H 2007 Messenger RNA in oocytes and embryos in relation to embryo viability. Theriogenology 68 S77-S83. (doi:10.1016/j.theriogenology.2007.04.028)

Yuan YQ, van Soom A, Leroy JLMR, Dewulf J, van Zeveren A, de Kruif A \& Peelman LJ 2005 Apoptosis in cumulus cells, but not in oocytes, may influence bovine embryonic developmental competence. Theriogenology 63 2147-2163. (doi:10.1016/j.theriogenology. 2004.09.054)

Received 19 November 2010

First decision 23 December 2010

Revised manuscript received 25 February 2011

Accepted 17 March 2011 